\title{
FLUORESCENCE BLIND STRUCTURED ILLUMINATION MICROSCOPY: A NEW RECONSTRUCTION STRATEGY
}

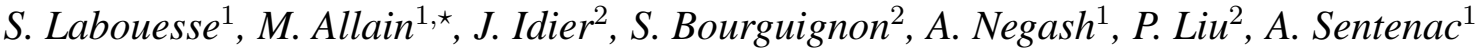 \\ ${ }^{1}$ Aix-Marseille Université, CNRS, Centrale Marseille, Institut Fresnel (France) \\ ${ }^{2}$ École Centrale de Nantes, CNRS, IRCCyN (France) \\ * Corresponding author (marc.allain@fresnel.fr)
}

\begin{abstract}
In this communication, a fast reconstruction algorithm is proposed for fluorescence blind structured illumination microscopy (SIM) under the sample positivity constraint. This new algorithm is by far simpler and faster than existing solutions, paving the way to $3 \mathrm{D}$ and real-time $2 \mathrm{D}$ reconstruction.
\end{abstract}

Index Terms - Super-resolution, fluorescence microscopy, speckle imaging, near-black object model, proximal splitting.

\section{INTRODUCTION}

Classical wide-field fluorescence microscopy aims at imaging the fluorescence density $\rho$ emitted from a marked biological sample. In the linear regime, the recorded intensity is related to $\rho$ via a simple convolution model [9]. If one proceeds to $M$ distinct acquisitions, the dataset $\left\{y_{m}\right\}_{m=1}^{M}$ is given by

$$
y_{m}=h \otimes\left(\rho \times I_{m}\right)+\varepsilon_{m} \quad m=1 \cdots M
$$

where $\otimes$ is the convolution operator, $h$ is the point-spread function (PSF), $I_{m}$ is the $m$-th illumination intensity pattern, and $\varepsilon_{m}$ is a perturbation term accounting for (electronic) noise in the detection and model errors. The final resolution of the microscope is ultimately limited by the optical transfer function (OTF) whose cutoff frequency is fixed by the emitted wavelength and by the numerical aperture of the microscope objective. However, if any frequency component above this limit cannot be measured with uniform illuminations, structured illuminations can be used to shift high-frequency components of the object into the OTF support [11]. Such a strategy results in the standard structured illumination microscopy (SIM) that resorts to harmonic illumination patterns to achieve super-resolution reconstruction. Because SIM uses the illumination patterns as references, strong artifacts are induced if the patterns are not known with sufficient accuracy [14, 2]. From a practical viewpoint, such a condition is very stringent and restricts standard SIM to thin samples or to samples with small refraction indices [14].
The Blind-SIM strategy [14] has been proposed to tackle this problem, the principle being to retrieve the sample fluorescence density without the knowledge of the illumination patterns, thereby extending the potential of SIM. In addition, this strategy promotes the use of speckle illumination patterns instead of harmonic illumination patterns, the latter standard case being much more difficult to generate. From the methodological viewpoint, Blind-SIM relies on the simultaneous reconstruction of the fluorescence density and of the illumination patterns. More precisely, in [14], joint reconstruction is achieved through the iterative resolution of a constrained least-squares problem based on conjugate gradient iterations. However, the computational time of such a scheme (as reported in [14, Supplementary material]) clearly restricts the applicability of the resulting joint blind-SIM strategy. In this paper, the implementation issues of joint Blind-SIM are revisited and drastically simplified: a much improved implementation is proposed, with an execution time decreased by several orders of magnitude. Moreover, it can be highly parallelized, opening the way to real-time reconstructions.

A few other contributions advocated the use of speckle patterns for super-resolved imaging in fluorescent [13, 15] or photo-acoustic [4] microscopy. Because these strategies are derived from the statistical modeling of the speckle, the resulting super-resolution strongly rests on the random nature of the illumination patterns [12]. In contrast, this communication stresses that the super-resolution mechanism behind joint blind-SIM relies on a sparsity and positivity constraint enforced by the illumination pattern. As a result, super-resolved reconstructions can be obtained with a wide range of illuminations patterns (even with deterministic illuminations) as soon as they cancel-out "frequently" the object.

\section{BLIND-SIM PROBLEM REFORMULATION}

In the sequel, we focus on a discretized formulation of the observation model (1). Solving the two-dimensional (2D) joint Blind-SIM reconstruction problem is equivalent to finding a joint solution $\left(\widehat{\boldsymbol{\rho}},\left\{\widehat{\boldsymbol{I}}_{m}\right\}_{m=1}^{M}\right)$ to the following constrained 
minimisation problem [14]:

$$
\begin{aligned}
\min _{\boldsymbol{\rho},\left\{\boldsymbol{I}_{m}\right\}} & \sum_{m=1}^{M}\left\|\boldsymbol{y}_{m}-\boldsymbol{H} \operatorname{diag}(\boldsymbol{\rho}) \boldsymbol{I}_{m}\right\|^{2} \\
\text { subject to } & \sum_{m} \boldsymbol{I}_{m}=M \times \boldsymbol{I}_{0} \\
\text { and } & \rho_{n} \geq 0, \quad I_{m ; n} \geq 0, \quad \forall m, n
\end{aligned}
$$

with $\boldsymbol{H} \in \mathbb{R}^{N \times N}$ the $2 \mathrm{D}$ convolution matrix built from the discretized PSF. We also denote $\boldsymbol{\rho}=\operatorname{vect}\left(\rho_{n}\right) \in \mathbb{R}^{N}$ the discretized fluorescence density, $\boldsymbol{y}_{m}=\operatorname{vect}\left(y_{m ; n}\right) \in \mathbb{R}^{N}$ the $m$-th recorded image, and $\boldsymbol{I}_{m}=\operatorname{vect}\left(I_{m ; n}\right) \in \mathbb{R}^{N}$ the $m$-th illumination with spatial mean $\boldsymbol{I}_{0}=\operatorname{vect}\left(I_{0 ; n}\right) \in \mathbb{R}^{N}$. Note that (2) is a biquadratic problem, which was tackled in [14] by a rather complex iterative scheme. However, problem (2) has a very specific structure that can benefit from a specific optimization strategy, which is explained hereafter.

\subsection{A reformulation of the joint blind-SIM strategy}

Let us first consider problem (2) without the equality constraint (2b). It then becomes equivalent to $M$ quadratic minimisation problems

$$
\begin{array}{ll} 
& \min _{\boldsymbol{q}_{m}}\left\|\boldsymbol{y}_{m}-\boldsymbol{H} \boldsymbol{q}_{m}\right\|^{2} \\
\text { subject to } & \boldsymbol{q}_{m} \geq 0
\end{array}
$$

with $\boldsymbol{q}_{m}:=\operatorname{vect}\left(\rho_{n} \times I_{m ; n}\right)$. Each minimisation problem (3) can be solved in a simple and efficient way (see Sec. 3), hence providing a set of global minimizers $\left\{\widehat{\boldsymbol{q}}_{m}\right\}_{m=1}^{M}$. Although the latter set corresponds to an infinite number of solutions $\left(\widehat{\boldsymbol{\rho}},\left\{\widehat{\boldsymbol{I}}_{m}\right\}_{m=1}^{M}\right)$, the equality constraint in (2b) defines a unique solution such that $\operatorname{diag}(\widehat{\boldsymbol{\rho}}) \times \widehat{\boldsymbol{I}}_{m}=\widehat{\boldsymbol{q}}_{m}$ for all $m$ :

$$
\begin{aligned}
\widehat{\boldsymbol{\rho}} & =\operatorname{diag}\left(\boldsymbol{I}_{0}\right)^{-1} \overline{\boldsymbol{q}} \\
\forall m \quad \widehat{\boldsymbol{I}}_{m} & =\operatorname{diag}(\widehat{\boldsymbol{\rho}})^{-1} \widehat{\boldsymbol{q}}_{m}
\end{aligned}
$$

where

$$
\overline{\boldsymbol{q}}:=\frac{1}{M} \sum_{m} \widehat{\boldsymbol{q}}_{m}=\operatorname{diag}(\widehat{\boldsymbol{\rho}}) \frac{1}{M} \sum_{m} \widehat{\boldsymbol{I}}_{m}=\operatorname{diag}(\widehat{\boldsymbol{\rho}}) \boldsymbol{I}_{0} .
$$

Moreover, the following implications hold:

$$
\begin{array}{r}
I_{0 ; n}>0, \quad \text { and } \quad \widehat{q}_{m ; n}>0, \\
\Longrightarrow \quad \widehat{I}_{m, n} \geq 0 \quad \text { and } \quad \widehat{\rho}_{n} \geq 0 \quad \forall n, m .
\end{array}
$$

Because we are dealing with intensity patterns, the condition $\boldsymbol{I}_{0} \geq 0$ is always met, hence ensuring the positivity of both the density and the illumination estimates. We also note that a solution defined by (4) exists as long as $I_{0 ; n} \neq 0$ and $\widehat{\rho}_{n} \neq 0$, $\forall n$. The first condition is met if the sample is illuminated everywhere, which is an obvious minimal requirement. For any pixel sample such that $\widehat{\rho}_{n}=0$, the corresponding illumination $\widehat{I}_{m ; n}$ is not defined; this is not a problem as long as the fluorescence density $\rho$ is the only quantity of interest.

\subsection{Toward a penalized joint blind-SIM strategy}

Whereas the mechanism that conveys super-resolution with known structured illuminations is well understood (see [10] for instance), the super-resolution capacity of joint blind-SIM has not been characterized yet. It can be made clear, however, that the positivity constraint (2c) plays a central role in this regard. Let $\boldsymbol{H}^{+}$be the pseudo-inverse of $\boldsymbol{H}$ [8, Sec. 5.5.4]. Then, any solution to the problem (2a)-(2b) [i.e., without positivity constraints] reads

$$
\begin{aligned}
\widehat{\boldsymbol{\rho}} & =\operatorname{diag}\left(\boldsymbol{I}_{0}\right)^{-1}\left(\boldsymbol{H}^{+} \overline{\boldsymbol{y}}+\overline{\boldsymbol{q}}^{\perp}\right) \\
\widehat{\boldsymbol{I}}_{m} & =\operatorname{diag}(\widehat{\boldsymbol{\rho}})^{-1}\left(\boldsymbol{H}^{+} \boldsymbol{y}_{m}+\boldsymbol{q}_{m}^{\perp}\right),
\end{aligned}
$$

with $\overline{\boldsymbol{y}}=\frac{1}{M} \sum_{m} \boldsymbol{y}_{m}, \quad$ and $\quad \overline{\boldsymbol{q}}^{\perp}=\frac{1}{M} \sum_{m} \boldsymbol{q}_{m}^{\perp}$ where $\boldsymbol{q}_{m}^{\perp}$ is an arbitrary element of the kernel of $\boldsymbol{H}$, i.e. with arbitrary frequency components above the OTF cutoff frequency. Hence, the formulation $(2 \mathrm{a})-(2 \mathrm{~b})$ has no capacity to discriminate the correct high frequency components, which means that it has no super-resolution capacity. Under the positivity constraint (2c), we thus expect that the super-resolution capacity of joint blind-SIM depends on the fact that each illumination pattern $\boldsymbol{I}_{m}$ activates the positivity constraint on $\boldsymbol{q}_{m}$ in a frequent manner. Such adequate illumination patterns can be easily generated as speckle images, as proposed by [14]. In contrast, standard SIM rests upon the amplitude modulation of the object, i.e., it only needs broad-band spectra illumination patterns.

Let us stress that each problem (3) is convex quadratic, and thus admits only global solutions, which in turn provide global solutions to problem (2), when recombined according to (4a)-4b). On the other hand, problems (3) may not admit unique solutions, since $\left\|\boldsymbol{y}_{m}-\boldsymbol{H} \boldsymbol{q}_{m}\right\|^{2}$ is not strictly convex in $\boldsymbol{q}_{m}$. A simple way to enforce unicity is to slightly modify (3) by adding a strictly convex penalization term. We are thus led to solving

$$
\min _{\boldsymbol{q}_{m} \geq 0} \sum_{m=1}^{M}\left\|\boldsymbol{y}_{m}-\boldsymbol{H} \boldsymbol{q}_{m}\right\|^{2}+\varphi\left(\boldsymbol{q}_{m}\right) .
$$

Another advantage of such an approach is that $\varphi$ can be chosen so that robustness to the noise is granted and/or some expected features in the solution are enforced. In particular, the analysis conveyed above suggests that favoring sparsity in each $\boldsymbol{q}_{m}$ is suited since speckle or periodic illumination patterns tend to frequently cancel or nearly cancel the components $q_{m ; n}$ within the product image $\boldsymbol{q}_{m}$. For such illuminations, the Near-Black Object introduced in Donoho's seminal paper [6] is an appropriate modeling and, following this line, we found that the standard separable $\ell_{1}$ penalty provides super-resolved reconstructions

$$
\varphi\left(\boldsymbol{q}_{m} ; \alpha, \beta\right):=\beta\left\|\boldsymbol{q}_{m}\right\|^{2}+\alpha \sum_{n}\left|q_{m ; n}\right|,
$$

with $\alpha \geq 0$ and $\beta>0$ some hyper-parameters. With properly tuned $(\alpha, \beta)$, our penalized joint Blind-SIM strategy is expected to bring super-resolution if "adequate" illumination patterns are used, i.e., they need to locally cancel out the imaged sample and their average $\boldsymbol{I}_{0}$ has to be known. 


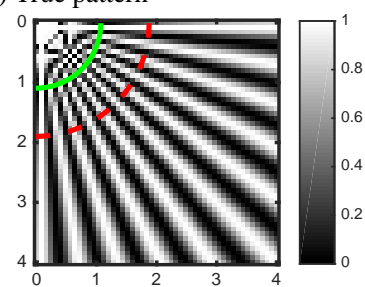

(c) Blind-SIM (periodic patterns)

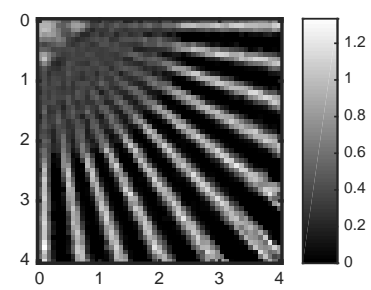

(b) Dec. of $\overline{\boldsymbol{y}}=\sum_{m} \boldsymbol{y}_{m} / M$

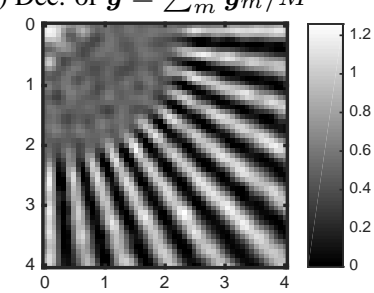

(d) Blind-SIM (speckle patterns)

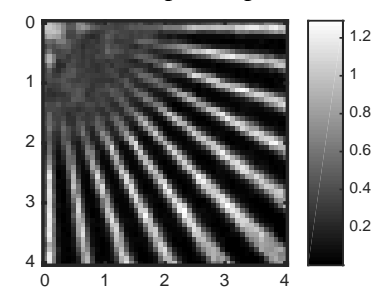

Fig. 1. Right lower quadrant of the $(100 \times 100$ pixels $)$ true fluorescence pattern (a), positive deconvolution of the averaged speckle patterns (b), and penalized joint blind-SIM reconstructions with $M=9$ periodic (c) and $M=200$ speckle patterns (d). The graduations are in $\lambda$. The dashed (resp. solid) lines in (a) corresponds to the spatial frequencies transmitted by the OTF support (resp. twice the OTF support).

\subsection{Numerical illustration}

For illustrative purposes, the numerical blind-SIM experiment presented in [14] is now considered. The ground-truth consists in the 2D 'star-like' fluorescence pattern depicted in Fig. 1 a). The $M$ collected images are simulated following (1) with the PSF $h$ given by the Airy pattern that reads in polar $(r, \theta)$ coordinates

$$
h(r, \theta)=\left(\frac{J_{1}\left(r k_{0} \mathrm{NA}\right)}{k_{0} r}\right)^{2} \frac{k_{0}^{2}}{\pi}
$$

where $J_{1}$ is the first order Bessel function of the first kind, NA is the objective numerical aperture set to 1.49 , and $k_{0}=2 \pi / \lambda$ is the free-space wavenumber with $\lambda$ the fluorescence emission and the excitation wavelength. The image sampling step for all the simulations is set to $\lambda / 12$. The illumination set $\left\{\boldsymbol{I}_{m}\right\}_{m=1}^{M}$ consists either in $M=9$ periodic patterns with spatial frequency equal to $2.4 / \lambda$, or in $M=200$ speckle patterns with spatial correlation given by (8). Finally, the collected images are corrupted with Gaussian noise. The standard deviation for a single acquisition was chosen so that the total SNR is $40 \mathrm{~dB}$ for both the periodic and speckle experiments. The subproblem hyperparameters were empirically set to $\left(\alpha=0.3, \beta=10^{-4}\right)$ for both the periodic and the speckle illuminations. The reconstructions of Fig. 1(c)-(d) clearly show a super-resolution effect similar to the one obtained in [14]. In particular, this simulation corroborates the empirical statement that $M \approx 10$ harmonic illuminations and $M \approx 200$ speckle illuminations produce almost equivalent

super-resolved reconstructions. Obviously, imaging with random speckle patterns remains an attractive strategy since it is achieved with a very simple experimental setup, see [14] for details. The blind-SIM reconstructions shown in Fig. 11were produced with 1000 iterations of the algorithm proposed in Sec. 3, the total computation time for the processing of the $(M=200)$ speckle and $(M=9)$ harmonic patterns is 360 and 17 seconds with a standard Matlab implementation on a regular computer. On the other hand, let us remark that our strategy requires an explicit tuning of the parameters $\alpha$ and $\beta$, whereas the constrained conjugate gradient approach proposed in [14] is regularized through the number of iterates. Since adequate values of $\alpha$ and $\beta$ will depend mostly on experimental parameters (PSF, noise and signal levels, number of views), a simple calibration step seems possible.

\section{A NEW OPTIMIZATION STRATEGY}

We now consider the algorithmic issues involved in the constrained optimization problem (6)-(7). For the sake of simplicity, the subscript $m$ in $\boldsymbol{y}_{m}$ and $\boldsymbol{q}_{m}$ are dropped, however, the reader should keep in mind that the algorithm presented in the next section only aims at solving one of the $M$ subproblems involved in the final joint Blind-SIM reconstruction. At first, let us note that our minimization problem (6)-(7) is an instance of the more general statement

$$
\min _{\boldsymbol{q} \in \mathbb{R}^{N}}[f(\boldsymbol{q}):=g(\boldsymbol{q})+h(\boldsymbol{q})]
$$

where $g$ and $h$ are closed-convex functions that may not share the same regularity assumptions: $g$ is supposed to be a smooth function with a $L$-Lipschitz continuous gradient $\nabla g$, but $h$ needs not to be smooth. Our penalized joint Blind-SIM problem hence takes the form of (9) with

$$
\begin{aligned}
& g(\boldsymbol{q})=\|\boldsymbol{y}-\boldsymbol{H} \boldsymbol{q}\|^{2}+\beta\|\boldsymbol{q}\|^{2} \\
& h(\boldsymbol{q})=\alpha \sum_{n} \phi\left(q_{n}\right)
\end{aligned}
$$

where $\phi: \mathbb{R} \rightarrow \mathbb{R} \cup\{+\infty\}$ is such that

$$
\phi(u):= \begin{cases}u & \text { if } u \geq 0 . \\ +\infty & \text { elsewhere. }\end{cases}
$$

Constrained non-smooth optimization problems (9) can be solved by a dedicated proximal iteration. For that purpose, FISTA [3] is recognized as a fast and numerically efficient alternative: let $\boldsymbol{q}^{(0)}$ be a feasible initial-guess and $\boldsymbol{\omega}^{(0)}=\boldsymbol{q}^{(0)}$, the FISTA update reads

$$
\begin{aligned}
& \boldsymbol{q}^{(k+1)} \longleftarrow \mathcal{P}\left(\boldsymbol{\omega}^{(k)}-\gamma \nabla g\left(\boldsymbol{\omega}^{(k)}\right)\right) \\
& \boldsymbol{\omega}^{(k+1)} \longleftarrow \boldsymbol{q}^{(k+1)}+\frac{k-1}{k+2}\left(\boldsymbol{q}^{(k+1)}-\boldsymbol{q}^{(k)}\right)
\end{aligned}
$$

with $\gamma>0$ and $\mathcal{P}$ the proximity operator (or Moreau envelope) of the function $\gamma h$ that is easily found from $10 \mathrm{~b}-\mathrm{c}$ )

$$
\mathcal{P}(\boldsymbol{\omega})=\operatorname{vect}\left(\max \left\{\omega_{n}-\gamma \alpha, 0\right\}\right) .
$$



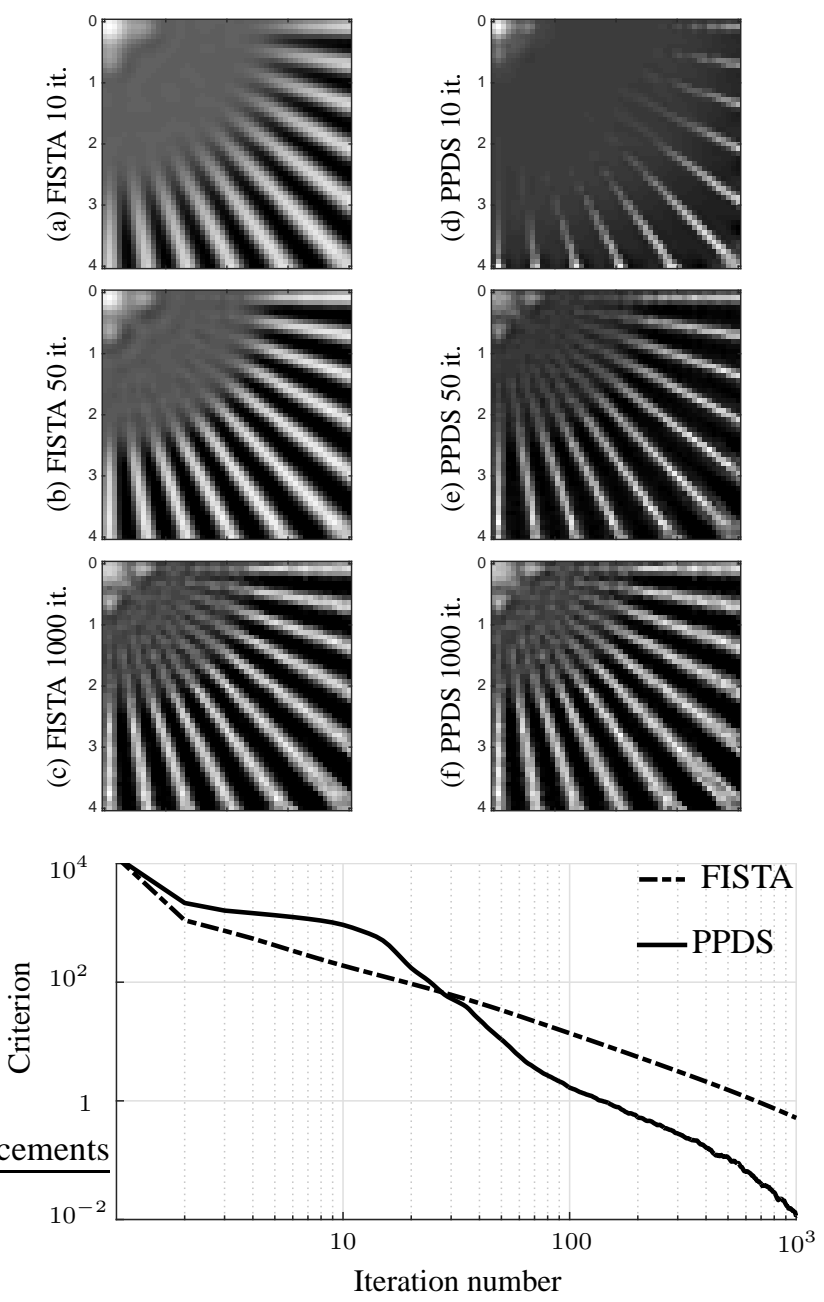

Fig. 2. [Top] Harmonic joint blind-SIM reconstruction of the fluorescence pattern achieved by the minimization of the criterion (6) with 10,50 or 1000 FISTA (abc) or PPDS (def) iterations. [Bottom] Criterion value (log-log scale) as a function of the PPDS (plain) or FISTA (dot) iteration number. All these simulations were performed with $\left(\alpha=0.3, \beta=10^{-4}\right)$.

Global convergence of (11) is granted provided that the stepsize is such that $0<\gamma<1 / L$ with $L=2\left(\lambda_{\max }\left(\boldsymbol{H}^{t} \boldsymbol{H}\right)+\beta\right)$ where $\lambda_{\max }(\boldsymbol{A})$ denotes the highest eigenvalue of the matrix $\boldsymbol{A}$. The decreasing rate achieved by $f\left(\boldsymbol{q}^{(k)}\right)$ is $O\left(1 / k^{2}\right)$, which is a substantial gain compared to the $O(1 / k)$ rate of the standard proximal iteration [3]. For our specific problem, however, the convergence speed of FISTA is too low. Figure 2 gives an illustration of this issue: FISTA requires almost one thousand iterations to converge. This is precisely the motivation for the introduction of the preconditioned primal-dual splitting (PPDS), a new algorithm that rests on the very versatile splitting technique proposed in [5]. Due to space limits, however, the comprehensive presentation of this iterative strategy is not detailed. Let $(\tau, \rho, \sigma)$ be a triplet of positive values, the PPDS iteration consists in a primal-dual update that reads

$$
\begin{aligned}
& \boldsymbol{q}^{(k+1)} \longleftarrow \boldsymbol{q}^{(k)}-\rho \tau \boldsymbol{B} \boldsymbol{\zeta}^{(k)} \\
& \boldsymbol{\omega}^{(k+1)} \longleftarrow \boldsymbol{\omega}^{(k)}+\rho\left[\mathcal{P}_{\star}\left(\boldsymbol{\delta}^{(k)}\right)-\boldsymbol{\omega}^{(k)}\right]
\end{aligned}
$$

with $\boldsymbol{\zeta}^{(k)}:=\boldsymbol{\nabla} g\left(\boldsymbol{q}^{(k)}\right)+\boldsymbol{\omega}^{(k)}$ and where the preconditioning matrix $\boldsymbol{B}$ is chosen from the Geman and Yang semi-quadratic construction [7], [1, Eq. (6)]

$$
\boldsymbol{B}:=\left(2 \boldsymbol{H}^{t} \boldsymbol{H}+2 \beta \boldsymbol{I} / a\right)^{-1}
$$

with $a>0$ a free parameter. In the dual update (13b), we noted $\boldsymbol{\delta}^{(k)}:=\boldsymbol{\omega}^{(k)}+\sigma\left(\boldsymbol{q}^{(k)}-2 \tau \boldsymbol{B} \boldsymbol{\zeta}^{(k)}\right)$ and

$$
\mathcal{P}_{\star}(\boldsymbol{\omega})=\operatorname{vect}\left(\min \left\{\omega_{n}, \alpha\right\}\right) .
$$

The convergence of the sequence defined by (13) is granted if some conditions are met for the parameters $(\rho, \tau, \sigma, a)$. Within the convergence domain ensured by [5, Theorem 5.1], the practical tuning of these parameters is somewhat tricky as it may dramatically impair the convergence speed. An automatic and efficient tuning strategy ensuring convergence has been devised; the PPDS reconstructions shown in Figure 2 were obtained with this tuning. The interest of the proposed strategy is that the iteration (13) can be easily implemented at a low computational cost. Indeed, since $\nabla g$ is a linear function, the primal step (13a) can be transposed in the Fourier domain, and it is easy to show that the computational burden of the PPDS iteration is dominated by only one FFT/iFFT pair. In other words, for our problem (10), FISTA and PPDS share the same complexity per iteration.

\section{CONCLUSION}

The reformulation presented in Sec. 2 unveiled some of the super resolution properties of the joint reconstruction problem introduced in [14]. We feel however that this joint blind-SIM approach deserves further investigations, both from the theoretical and the experimental viewpoints. In particular, one expected difficulty arising in the processing of real data sets is the strong background induced in the focal plane by the out-of-focus light. This phenomenon prevents the local extinction of the excitation intensity, hence destroying the expected super-resolution in joint blind-SIM. The modeling of this background with a very smooth function is possible [16] and will be considered. A different approach would be to solve the reconstruction problem in its 3D structure, which is numerically challenging, but remains a mandatory step to achieve 3D reconstructions. Finally, the PPDS strategy introduced in this communication is a promising optimization tool that will be tested with other applications. In particular, the deconvolution under a positivity constraint for the standard SIM problem should be considered shortly.

Acknowledgement. The GDR ISIS and the Agence Nationale de la Recherche (ANR-12-BS03-0006) are acknowledged for the partial funding of this work. 


\section{REFERENCES}

[1] M. Allain, J. Idier, and Y. Goussard. On global and local convergence of half-quadratic algorithms. IEEE Transactions on Image Processing, 15(5):1130-1142, May 2006.

[2] R. Ayuk, H. Giovannini, A. Jost, E. Mudry, J. Girard, T. Mangeat, N. Sandeau, R. Heintzmann, K. Wicker, K. Belkebir, and A. Sentenac. Structured illumination fluorescence microscopy with distorted excitations using a filtered blind-SIM algorithm. Optics Letters, 38(22):4723-4726, Nov 2013.

[3] A. Beck and M. Teboulle. A fast iterative shrinkagethresholding algorithm for linear inverse problems. SIAM J. Imaging Sciences, 2(1):183-202, 2009.

[4] T. Chaigne, J. Gateau, M. Allain, O. Katz, S. Gigan, A. Sentenac, and E. Bossy. Super-resolution photoacoustic fluctuation imaging with multiple speckle illumination. Optica, 3(1):54-57, Jan 2016.

[5] L. Condat. A primal-dual splitting method for convex optimization involving Lipschitzian, proximable and linear composite terms. J. Optimization Theory and Applications, 158(2):460-479, August 2013.

[6] D. L. Donoho, A. M. Johnstone, J. C. Hoche, and A. S. Stern. Maximum entropy and the nearly black object. Journal of the Royal Statistical Society, 198:41-81, 1992.

[7] D. Geman and C. Yang. Nonlinear image recovery with half-quadratic regularization. IEEE Transactions on Image Processing, 4(7):932-946, July 1995.

[8] G. H. Golub and C. H. Van Loan. Matrix computation. The Johns Hopkins University Press, Baltimore, 3rd ed. edition, 1996.

[9] J.W. Goodman. Introduction to Fourier Optics. Roberts \& Company Publishers, 2005.

[10] M. G. L. Gustafsson. Surpassing the lateral resolution limit by a factor of two using structured illumination microscopy. Journal of Microscopy, 2000.

[11] R. Heintzmann and C. Cremer. Laterally modulated excitation microscopy: improvement of resolution by using a diffraction grating. In Proc. SPIE, Optical Biopsies and Microscopic Techniques III, pages 185-196, 1999.

[12] J. Idier, S. Labouesse, P. Liu, M. Allain, S. Bourguignon, and A. Sentenac. A theoretical analysis of the super-resolution capacity of imagers using speckle illuminations. Research rep., IRCCyN/Institut Fresnel, 2015.
[13] J. Min, J. Jang, D. Keum, S.-W. Ryu, C. Choi, K.-H. Jeong, and J. C. Ye. Fluorescent microscopy beyond diffraction limits using speckle illumination and joint support recovery. Scientific reports, 3, 2013.

[14] E. Mudry, K. Belkebir, J. Savatier, E. Le Moal, C. Nicoletti, M. Allain, and A. Sentenac. Structured illumination microscopy using unknown speckle patterns. $\mathrm{Na}$ ture Photonics, 6:312-315, 2012.

[15] J. Oh, Y.-W. Cho, G. Scarcelli, and Y.-H. Kim. Subrayleigh imaging via speckle illumination. Opt. Lett., 38(5):682-684, Mar 2013.

[16] F. Orieux, E. Sepulveda, V. Loriette, B. Dubertret, and J.-C. Olivo-Marin. Bayesian estimation for optimized structured illumination microscopy. IEEE Transactions on image processing, 21(2):601-614, 2012. 